\section{Check for updates}

Cite this: Soft Matter, 2019, 15,8279

Received 28th June 2019

Accepted 3rd October 2019

DOI: $10.1039 / \mathrm{c} 9 \mathrm{sm} 01297 \mathrm{~d}$

rsc.li/soft-matter-journal

\title{
Using optical tweezing to control phase separation and nucleation near a liquid-liquid critical point $\dagger$
}

\author{
Finlay Walton (D) and Klaas Wynne (D)*
}

\begin{abstract}
About 20 years ago, it was shown that lasers can nucleate crystals in super-saturated solutions and might even be able to select the polymorph that crystallises. However, no theoretical model was found explaining the results and progress was slowed down. Here we show that laser-induced nucleation may be understood in terms of the harnessing of concentration fluctuations near a liquid-liquid critical point using optical tweezing in a process called laser-induced phase separation (LIPS) and LIPS and nucleation (LIPSaN). A theoretical model is presented based on the regular solution model with an added term representing optical tweezing while the dynamics are modelled using a Kramers diffusion equation, and the roles of heat diffusion and thermophoresis are evaluated. LIPS and LIPSaN experiments were carried out on a range of liquid mixtures and the results compared to theory.
\end{abstract}

\section{Introduction}

In the late 1990s and early 2000s, it was shown that a nanosecond laser can be used to induce nucleation of crystals in a supersaturated solution through a non-photochemical process. ${ }^{1}$ Most excitingly, it was reported that the laser polarisation could be used to control which polymorph would nucleate, promising an unprecedented degree of control. ${ }^{2,3}$ Subsequent work showed that laser pulses can induce nucleation of various crystals, ${ }^{4-8}$ liquid crystals $^{9,10}$ and bubbles. ${ }^{11}$ However, these nanosecond-laser nucleation experiments defied all explanation. ${ }^{12-14}$

A series of publications has shown that optical tweezing can be used to nucleate crystals from super-saturated ${ }^{15-18}$ and strangely even from under-saturated solutions. ${ }^{19}$ Optical tweezing is a technique used widely in physics and biology and involves the trapping of a high-refractive-index particle through optical forces. ${ }^{20}$ Thus, it was assumed that in these experiments the laser was tweezing pre-existing clusters or pre-nuclei. ${ }^{16}$ However, these optical tweezing nucleation experiments were only shown to work on a liquid-gas interface. This strongly suggests that heating, evaporation, convection, and Marangoni effects play critical roles, ${ }^{21}$ which would also explain why the method works in under-saturated solutions. However, very excitingly, these experiments also demonstrated polymorph selection. ${ }^{15-19}$ As in the case of non-photochemical laser-induced nucleation, no sensible theory is available to describe the physics of these results. Thus, it is fair to say that a physical understanding of all of these phenomena is still sorely lacking.

School of Chemistry, University of Glasgow, UK.

E-mail: klaas.wynne@glasgow.ac.uk

$\dagger$ Electronic supplementary information (ESI) available. See DOI: 10.1039/c9sm01297d
A possible explanation for these laser-induced nucleation experiments might come from thermodynamics. According to classical Gibbs nucleation theory, nucleation is impeded by the fact that a growing nucleus has an energetically-unfavourable interface that results in a barrier on the way to the crystalline state. $^{22,23}$ This means that in a supersaturated solution or supercooled liquid, the crystal is the thermodynamically most stable state but-in the absence of heterogeneous nucleation sites - can only be accessed through random fluctuations leading to a nucleus exceeding a critical size. A number of recent experiments have called into question the validity of classical Gibbs nucleation theory and invoked the presence of so-called pre-nucleation clusters. ${ }^{24-26}$ Although there have been experimental studies reporting pre-nucleation clusters, they are still considered controversial. ${ }^{27,28}$

However, an older paper by Frenkel on protein crystallisation showed that nucleation is sped up, not simply by increasing the concentration, but by choosing a concentration that is near a (hidden) liquid-liquid demixing critical point. ${ }^{29-31}$ This is because critical concentration fluctuations give rise to liquidlike protein droplets (with a very high protein concentration) that increase the probability of the formation of a critical nucleus. The metastable critical point idea found some traction in the chemical engineering community where it was related to the phenomenon of "oiling out" without too much detailed theoretical analysis. ${ }^{23,32-34}$ Could liquid-liquid phase separation, oiling out, pre-nucleation clusters, and laser-induced nucleation all be aspects of the same phenomenon (26,35,36 $^{2}$

On approaching a liquid-liquid demixing critical point, concentration fluctuations increase. As a result, it should be easier for an external "force", such as optical tweezing, to manipulate the concentration locally. ${ }^{37-39}$ Optical tweezing 
normally involves the trapping of a high-refractive-index particle by the small forces exerted by a focused laser beam. However, a chemist's view of nucleation is like a chemical reaction where the supersaturated solution is the reactant state and the crystal (nucleus) the product state. Switching on an optical tweezing laser will lower the free energy of the product state (even if the crystal nucleus does not exist yet), will therefore increase the driving force and lower the barrier for the nucleation process, and increase the "reaction rate". This simple but novel idea was first tested by us on liquid mixtures. ${ }^{40,41}$

Here we will present a simple theoretical model of a liquid mixture based on the regular solution model ${ }^{42}$ including an extra term representing the stored electromagnetic energy of a tweezing laser. This model shows that the concentrationdependent free energy is indeed more easily perturbed near a critical point. ${ }^{40,41}$ Experiments were carried out on a number of mixtures-exhibiting an upper consolute point near room temperature-using a simple continuous wave (CW) diode laser that showed that the optical tweezer can pull the high refractiveindex liquid out of the mixture in a process named laserinduced phase separation (LIPS). ${ }^{40,41}$ Near the liquid-liquid binodal the laser can trigger the formation of phase-separated droplets through LIPS and nucleation (LIPSaN).

\section{Theory}

\section{Regular solution model of mixing}

In order to model the free energy associated with the mixing (and demixing) of two liquids, the widely used regular solution model will be employed. ${ }^{42}$ Define a liquid mixture with mole fractions of molecule $\mathrm{A}$ and $\mathrm{B}$ given by $x_{0}$ and $x_{\mathrm{B}}$, such that $x_{\mathrm{B}}=1-x_{0}$. The regular solution model defines the strength of the energetic interaction between $\mathrm{A}$ and $\mathrm{B}$ relative to their selfinteraction by

$$
\zeta=\frac{z}{2}\left(2 \varepsilon_{\mathrm{AB}}-\varepsilon_{\mathrm{AA}}-\varepsilon_{\mathrm{BB}}\right)
$$

where $z$ is the number of neighbours of each molecule in the mixture. The expression for the molar free energy of mixing (in $\mathrm{J} \mathrm{mol}^{-1}$ ) is then given by ${ }^{41}$

$$
F_{\text {mix }}\left(x_{0}, T, \zeta\right)=R T\left(x_{0} \ln x_{0}+x_{\mathrm{B}} \ln x_{\mathrm{B}}\right)+\zeta x_{0} x_{\mathrm{B}} .
$$

Here $z \varepsilon_{\mathrm{AA}}$ is approximately equal to the heat of vaporisation, which ranges from 0 (at the gas-liquid critical point) to $\sim 40 \mathrm{~kJ} \mathrm{~mol}^{-1}$. So, for poorly mixing liquids, $\zeta$ is positive and on the order of a few $\mathrm{kJ} \mathrm{mol}^{-1}$. This equation can be used to calculate the free energy of mixing for reasonable parameters as discussed previously. ${ }^{40,41}$

The coexistence curve (binodal) is at $\mathrm{d} F_{\text {mix }} / \mathrm{d} x=0$ and the spinodal at $\mathrm{d}^{2} F_{\text {mix }} / \mathrm{d} x^{2}=0$, which are easily solved for $T$ as

$$
T_{\text {binodal }}=\frac{\zeta\left(1-2 x_{0}\right) R^{-1}}{\ln \left(1-x_{0}\right)-\ln \left(x_{0}\right)}
$$

and

$$
T_{\text {spinodal }}=2 x_{0} \zeta\left(1-x_{0}\right) R^{-1}
$$

One obtains a qualitative representation of, for example, the decane-nitrobenzene binodal for $\zeta \approx 5 \mathrm{~kJ} \mathrm{~mol}^{-1}$. Although the width and slight asymmetry of the experimental binodal (see Fig. 6) is not reproduced, this simple model will be sufficient for our purposes here. The main differences are a critical point at a higher mole fraction and quantitative changes in the position of the binodal and spinodal.

Before considering the effect of optical tweezing, one should first consider the free-energy changes in a small volume due to spontaneous concentration fluctuations. Define $V_{0}$ as the volume of the sample container and $x_{0}$ as the initial mole fraction of $\mathrm{A}$ in the mixture. Now consider a small volume $V_{\text {laser, which will contain the focal volume of the tweezing }}$ laser that will cause LIPS. If the mole fraction of $\mathrm{A}$ in this volume changes to $x_{\text {laser }}$ and the mole fraction in the remaining volume to $x_{\text {rest }}$, conserving the total amount of $\mathrm{A}$ and $\mathrm{B}$ implies

$$
x_{\text {rest }}=\frac{x_{0} V_{0}-x_{\text {laser }} V_{\text {laser }}}{V_{0}-V_{\text {laser }}}
$$

and the total free energy of the phase-separated system is

$$
F_{\text {sep }}\left(x_{\text {laser }}\right)=\frac{V_{\text {laser }}}{V_{0}} F_{\text {mix }}\left(x_{\text {laser }}\right)+\frac{V_{\text {rest }}}{V_{0}} F_{\text {mix }}\left(x_{\text {rest }}\right) .
$$

Shown in Fig. 1 is the change in free energy, $\Delta F=F_{\text {sep }}-F\left(x_{0}\right)$, associated with a change of concentration in $\mathrm{A}$ in the laser volume for three different initial mole fractions: $0.3,0.5$ (the critical mole fraction), and 0.7 at a temperature just above the critical temperature. It can be seen that in all cases (as expected of course) the free energy increases when the concentration in the laser volume is changed away from the equilibrium concentration. Near the critical point the free-energy potential is relatively flat giving rise to large spontaneous fluctuations in the local concentration.

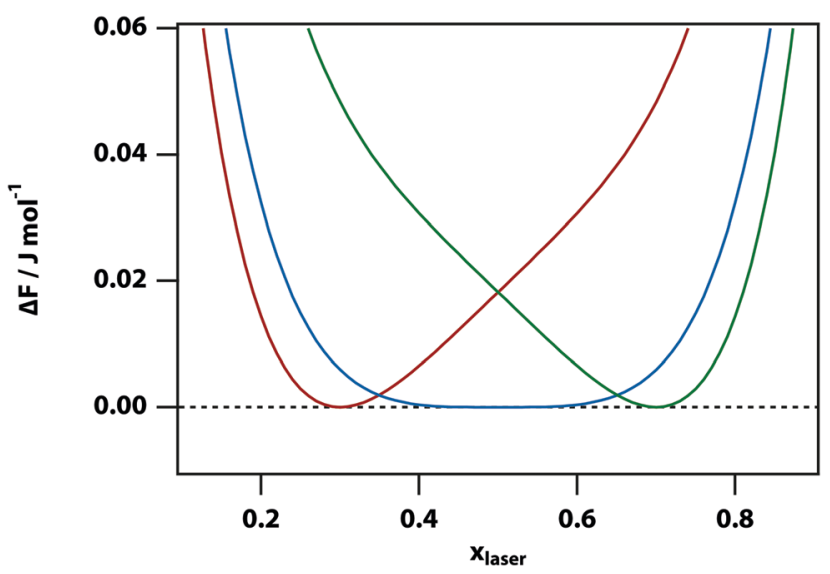

Fig. 1 The change in mixing free energy caused by changing the mole fraction in the laser volume. For $\zeta=5 \mathrm{~kJ} \mathrm{~mol}^{-1}, V_{\text {laser }}=10^{-3} \times V_{0}$, and $T=301 \mathrm{~K}$ (for these parameters, the liquid-liquid critical point is at $x=0.5$ and $T=300.68 \mathrm{~K}$ ) and the initial mole fractions are set to $x_{0}=0.3$ (red), 0.5 (blue), and 0.7 (green). 


\section{Adding in the effect of optical tweezing}

It is reasonable to assume that the refractive index in a mixture is approximately given by the concentration-weighted refractive indices of the component liquids $\mathrm{A}$ and $\mathrm{B}$, that is,

$$
n(x)=x n_{\mathrm{A}}+(1-x) n_{\mathrm{B}},
$$

which only minimally deviates from, e.g., the Lorentz-Lorenz law. As molecules are much smaller than the wavelength of light, it is reasonable to use the dipole approximation to calculate the stored electromagnetic energy, which is then given by $U_{\text {dip }}=-\varepsilon_{0} n^{2} E^{2}$ and thus the total stored electromagnetic energy in the laser volume is ${ }^{43,44}$

$$
U_{\text {laser }}=-\varepsilon_{0} n^{2}\left(x_{\text {laser }}\right) E^{2} V_{\text {laser }}
$$

The total change in free energy, including the effect of the laser, is then given by

$$
\begin{aligned}
\Delta F_{\text {sep }, \text { laser }}\left(x_{\text {laser }}\right)= & F_{\text {sep }}\left(x_{\text {laser }}\right)-F_{\text {mix }}\left(x_{0}\right) \\
& -\left[n^{2}\left(x_{\text {laser }}\right)-n^{2}\left(x_{0}\right)\right] \varepsilon_{0} E^{2} V_{\text {laser }} .
\end{aligned}
$$

The laser intensity in this expression, can be expressed as

$$
I=\varepsilon_{0} E^{2}=U_{\text {laser,ext }} / A c,
$$

where $U_{\text {laser,ext }}$ is the energy flux of the laser beam (in units of Watt, measured outside of the sample), $A$ is the area of the laser focus, and $c$ the speed of light. For a $50 \mathrm{~mW}$ laser beam (comparable to what was used in our experiments) focussed to a $1.7 \mu \mathrm{m}$ radius spot, this gives $I=\sim 1 \mathrm{~kJ} \mathrm{~m}^{-3}$.

The change of the free-energy potential by the optical tweezing effect is illustrated in Fig. 2 under the assumption that liquid B has the higher refractive index. Switching on the optical-tweezing potential biases the potential towards the right and therefore the laser volume is expected to become enriched with the high refractive index liquid. This process we will refer to as laser-induced phase separation (LIPS). ${ }^{40,41}$ In the metastable case, switching on the laser causes the system to tip from metastable (two minima separated by a barrier) to unstable (single minimum determined by the tweezing laser) at sufficient laser power, thereby triggering phase separation.

In the high temperature regime, the free-energy difference curve, eqn (9), has only a single minimum. Thus, in principle, one could find this stable minimum by determining $\mathrm{d} \Delta F_{\text {sep,laser }} /$ $\mathrm{d} x=0$, however, this does not have a simple analytical solution. Therefore, instead we determined the minimum of the free energy difference using a numerical algorithm. In the low temperature limit (below the spinodal), the free energy difference also has single minimum. However, for temperatures between the binodal and spinodal, the free energy difference has two minima. Our numerical approach implies that the metastable minimum will be missed and only the stable minimum will be found.

The experiments described below are carried out by using phase-contrast microscopy, which is sensitive to changes in refractive index. Since the refractive index is linearly proportional to the volume fraction in our model (as per eqn (7)), the volume fraction can be used as a proxy for the signal measured in the
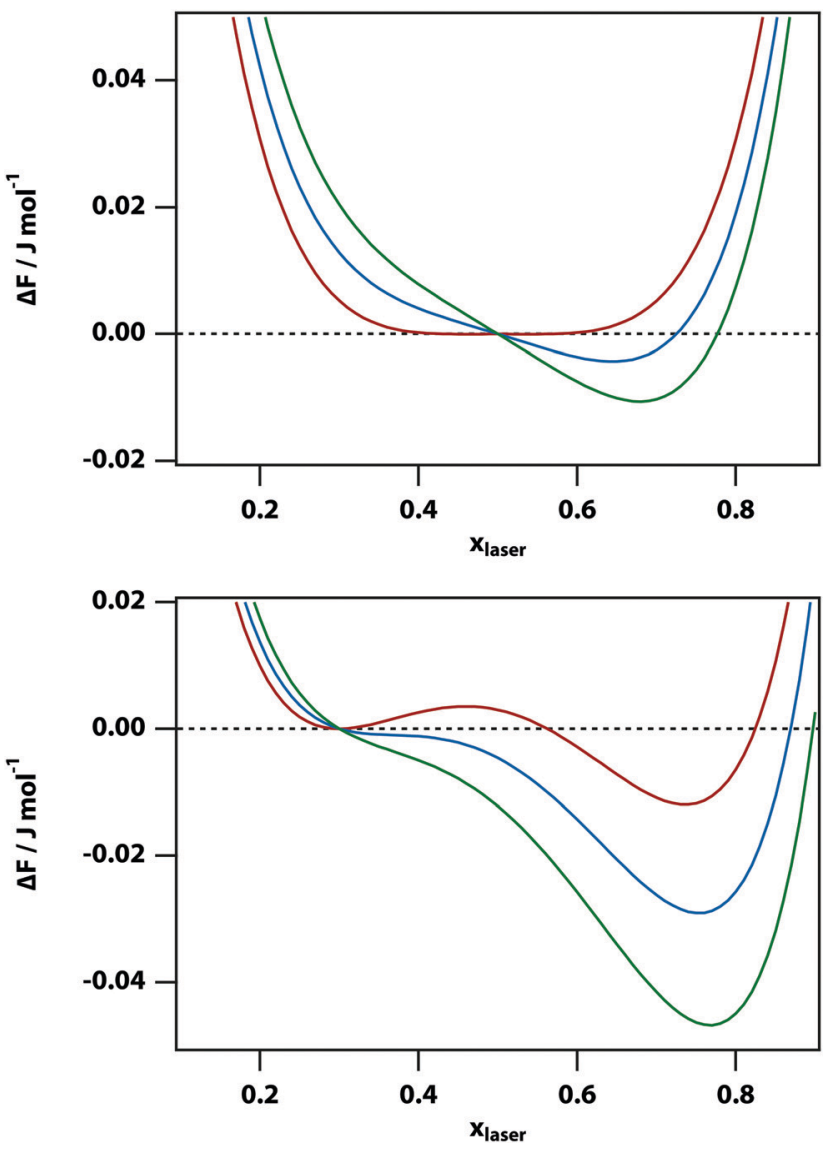

Fig. 2 Plots of the change in free energy $\Delta F$ in a nitrobenzene-decane mixture of mole fraction $x_{0}$ of nitrobenzene when the mole fraction is changed to $x_{\text {laser }}$ in a small volume. Here the initial nitrobenzene mole fraction is $x_{0}=0.5$ (top) and $x_{0}=0.3$ (bottom), the laser intensity is $I=0$ (red), 100 (blue), and 200 (green) $\mathrm{J} \mathrm{m}^{-3}, \zeta=5 \mathrm{~kJ} \mathrm{~mol}^{-1}$, and $T=300$ (top) and 280 (bottom) $\mathrm{K}$. The refractive indices are set to $n_{\text {decane }}=1.41$ and $n_{\text {nitrobenzene }}=1.54$. The panel at the top corresponds to the regime in which the two liquids are mixed and close to the liquid-liquid critical point. In the panel at the bottom, the system is metastable when the laser is off and the free energy could be lowered by phase separation after crossing a free-energy barrier.

experiments. Fig. 3 shows the predicted phase-contrast signal calculated by finding the $x$ that minimises the free-energy difference numerically and subtracting off the original volume fraction $x_{0}$, as a function of temperature, laser intensity, and initial mole fraction. At sufficiently high temperature all of these curves follow the power law $\left(T-T_{0}\right)^{-1}$ but deviate from this behaviour very near the binodal.

\section{The Kramers equation for diffusion}

The free-energy change induced by the laser (eqn (9)) gives rise to an optical trap that will draw in the liquid with the highest refractive index giving rise to LIPS kinetics. The liquid diffusion can be modelled by a Fokker-Planck equation, describing the evolution of the probability distribution function $p(x, t)$ of a random variable $x$ as

$$
\begin{aligned}
\frac{\partial}{\partial t} p(x, t)= & -\frac{\partial}{\partial x}[v(x, t) p(x, t)] \\
& +\frac{\partial^{2}}{\partial x^{2}}[D(x, t) p(x, t)]
\end{aligned}
$$



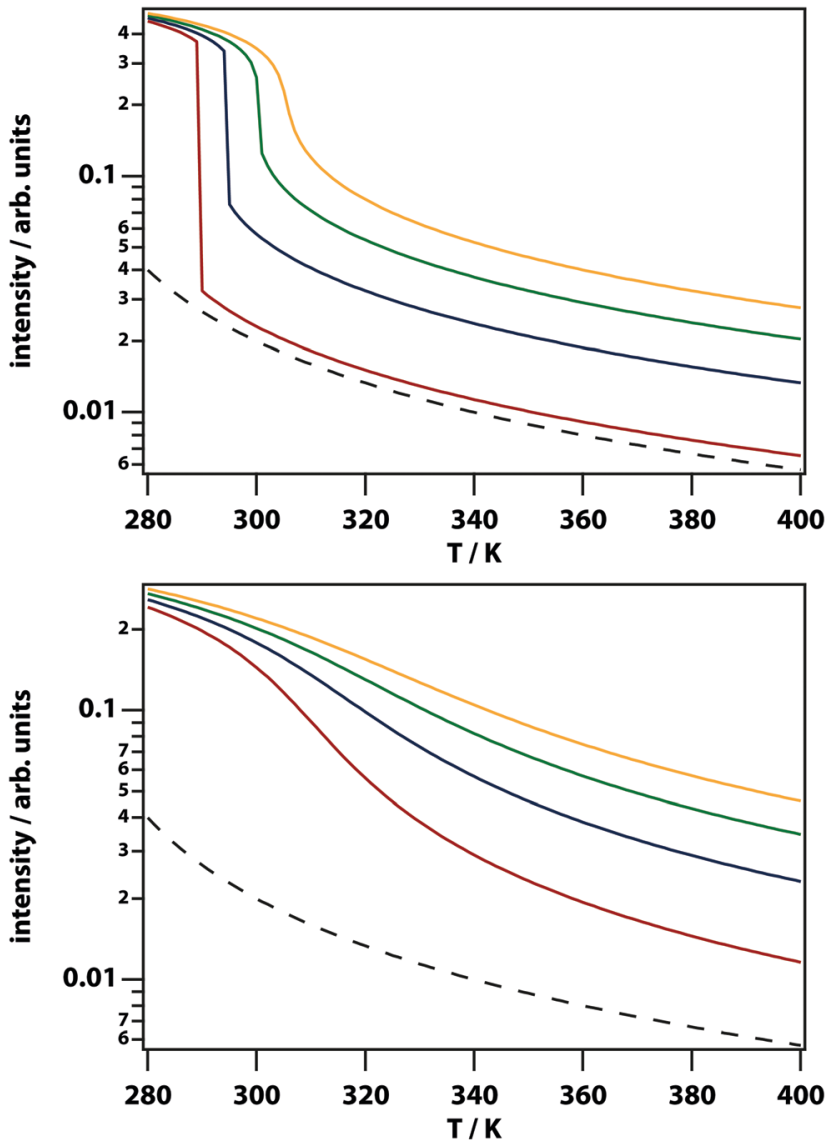

Fig. 3 Predicted phase-contrast microscopy signal strength as a function of laser intensity. Initial mole fraction is $x_{0}=0.3$ (top) and 0.5 (bottom), the laser intensity $I=100$ (red), 200 (blue), 300 (green), and 400 (yellow) $\mathrm{J} \mathrm{m}^{-3}$. Also shown is a power law $0.8(T-260)^{-1}$ (black dashed).

where $v(x, t)$ is the drift velocity and $D(x, t)$ the diffusion coefficient. In this case, the diffusion coefficient can be taken as constant and we will substitute $v(x)=-\mu \partial U(x) / \partial x$, that is, the drift velocity scales with the gradient of the free-energy trapping potential. Here

$$
\mu=\frac{1}{6 \pi \eta r}, \quad D=\frac{k_{\mathrm{B}} T}{6 \pi \eta r},
$$

$\eta$ is the viscosity, and $r$ the radius of the diffusing particle (a molecule in this case). Then it follows,

$$
\begin{aligned}
\frac{\partial}{\partial t} p(x, t)= & \frac{D}{k_{\mathrm{B}} T} \frac{\partial}{\partial x}\left[p(x, t) \frac{\partial U(x)}{\partial x}\right] \\
& +D \frac{\partial^{2}}{\partial x^{2}} p(x, t),
\end{aligned}
$$

which has been derived previously for the case of solvent-driven electron transfer. ${ }^{45}$

When the potential $U(x)$ is set to zero everywhere, one can easily derive the Green's function of this Fokker-Planck equation, which is given by

$$
p(x, t)=\frac{1}{\sqrt{4 \pi D t}} \mathrm{e}^{-x^{2} / 4 D t} .
$$

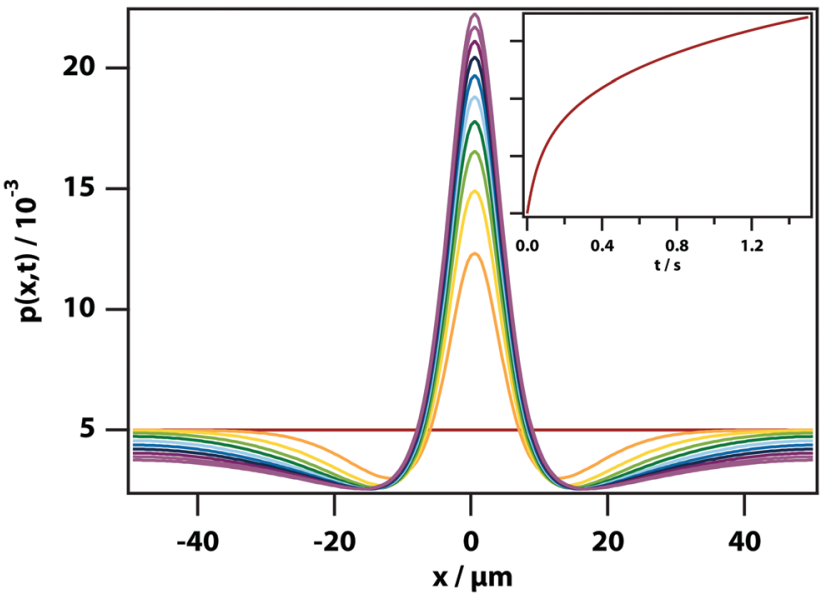

Fig. 4 Dynamics of LIPS. Simulation of the evolution of the concentration of the high refractive index substance under the influence of an optical tweezing force. Box length $100 \mu \mathrm{m}, 1 \mathrm{~ms}$ time step in simulation, 200 grid steps, $D_{\text {trans }}=3.1 \times 10^{-10} \mathrm{~m}^{2} \mathrm{~s}^{-1}, T=300 \mathrm{~K}, w=6 \mu \mathrm{m}$. (inset) The value on the peak as a function of time.

Thus, in the absence of a potential and in one dimension, the standard deviation of an initially delta-function distribution varies as $\sigma=\sqrt{2 D t}$. The diffusion coefficient $D$ can be approximated using the Stokes-Einstein expression eqn (12). If this equation is applied to nitrobenzene (molar mass $123.06 \mathrm{~g} \mathrm{~mol}^{-1}$, density $1.199 \mathrm{~g} \mathrm{~cm}^{-3}$, therefore $r=3.44 \mathrm{~nm}$, and $\eta=2.03 \mathrm{cP}$ ) one finds $D_{\text {trans }}=3.1 \times 10^{-10} \mathrm{~m}^{2} \mathrm{~s}^{-1}$.

In the case of a focussed laser with a Gaussian beam profile of width $w$, the trapping potential is simply

$$
U(x) \propto \mathrm{e}^{-x^{2} / 2 w^{2}}
$$

and the width is typically a few $\mu \mathrm{m}$ in our experiments (as long working distance objectives had to be used). The diffusion eqn (13) with the trapping potential eqn (15) was solved numerically using 2nd-order Runge-Kutta on a spatial grid. Typical results are shown in Fig. 4 with parameters chosen to be relevant to the nitrobenzene-hexadecane mixture. It can be seen that the trapping laser draws nitrobenzene into the focus leaving behind a depletion region that gradually fills in. The peak of the distribution grows on a timescale of $\sim 0.5 \mathrm{~s}$ determined by the diffusion coefficient $D$.

\section{Heat diffusion}

The trapping laser can also be partially absorbed by vibrational overtones resulting in a rise in temperature, which would counteract the optical-trapping effect. The one-dimensional heat equation is ${ }^{46}$

$$
\frac{\partial u}{\partial t}=\alpha\left(\frac{\partial^{2} u}{\partial x^{2}}\right)+\frac{1}{c_{\mathrm{p}} \rho} q,
$$

where $u(x, t)$ is the temperature field, $\alpha$ the thermal diffusivity (in $\mathrm{m}^{2} \mathrm{~s}^{-1}$ ) defined by $\alpha \equiv k /\left(c_{\mathrm{p}} \rho\right), k$ is the thermal conductivity, $c_{\mathrm{p}}$ is the specific heat capacity, $\rho$ is the mass density, and $q$ is the heat generated per unit volume (in $\mathrm{W} \mathrm{m}^{-3}$ ) by some external heat source (such as the laser). So, $\alpha$ in this expression 
has the same behaviour as $D$ in eqn (13). However, $\alpha \approx D \times 1000$, showing that heat diffuses $\sim 30 \times$ more rapidly (see eqn (14)).

As heat transport is rapid, it is reasonable to assume that the heat source is a delta function in space, which allows a stationary solution to be found if it is taken that heat is lost by cooling (for example, to the cooling stage used in our experiments). The stationary heat equation then becomes

$$
\frac{\partial u}{\partial t}=\alpha\left(\frac{\partial^{2} u}{\partial x^{2}}\right)+\frac{Q}{c_{\mathrm{p}} \rho} \delta(x)-\beta\left(u-T_{0}\right)=0,
$$

where $\delta(x)$ is the Dirac (in $=\mathrm{m}^{-1}$ ) delta function, $Q$ is the magnitude of the $\mathrm{CW}$ heat source (in $\mathrm{W} \mathrm{m}^{-2}$ ), $\beta$ is the rate of heat transfer (in $\mathrm{s}^{-1}$ ), and $T_{0}$ is the cooling-stage set temperature. This problem is easily solved in Fourier space, resulting in

$$
\tilde{u}=\frac{\frac{Q}{c_{\mathrm{p}} \rho}+\beta T_{0} 2 \pi \delta(k)}{\alpha k^{2}+\beta},
$$

which has poles at $k= \pm i \sqrt{\beta / \alpha}$. The inverse Fourier transform is solved by contour integration to yield

$$
u(x)=T_{0}+\Delta T \mathrm{e}^{-x / \sqrt{\alpha / \beta}},
$$

where

$$
\Delta T=\frac{1}{2} \sqrt{\frac{1}{\alpha \beta}}\left(\frac{Q}{c_{\mathrm{p}} \rho}\right) .
$$

The characteristic heating spot radius is therefore approximately $\sqrt{\alpha / \beta}$. The parameter $\beta$ can be calculated using Fourier's law of heat flow yielding

$$
\beta=\frac{\kappa_{\text {glass }}}{c_{\mathrm{p}, \text { liquid }} \rho d_{\text {liquid }} d_{\text {glass }}} .
$$

Using the thermal conductivity of glass $\left(\kappa_{\text {glass }} \cong 1 \mathrm{~W} \mathrm{~m}^{-1} \mathrm{~K}^{-1}\right)$, the specific heat capacity nitrobenzene $\left(c_{\mathrm{p} \text {,nitrobenzene }}=1400 \mathrm{~J} \mathrm{~kg}^{-1} \mathrm{~K}^{-1}\right)$, the mass density of nitrobenzene $\left(\rho_{\text {nitrobenzene }}=1175 \mathrm{~kg} \mathrm{~m}^{-3}\right)$, the thickness of the windows used in the experiments $\left(d_{\text {glass }}=80 \mu \mathrm{m}\right)$, it is found that $\beta=507 \mathrm{~Hz}$. Using the thermal diffusivity of nitrobenzene $^{47}\left(\alpha_{\text {nitrobenzene }}=0.94 \times 10^{-7} \mathrm{~m}^{2} \mathrm{~s}^{-1}\right)$ and eqn (20), we can calculate the stationary heating spot radius (at $1 / e$ of the maximum) as $\sqrt{ } \alpha / \beta=13 \mu \mathrm{m}$ for a glass window (and $\sqrt{ } \alpha / \beta=16 \mu \mathrm{m}$ for a mica window). This is significantly larger than the beam radius (see below), justifying the delta-function heat-source approximation. For a glass window, the absorbed laser power is $1.5 \mu \mathrm{W}$ out of $200 \mathrm{~mW}$, which leads to a maximum temperature rise of $1.6 \mathrm{~K}$ in the focus. This shows that sample heating is a nonnegligible effect at least at the highest laser powers used in our experiments.

\section{Thermophoresis}

In this work, we argue that LIPS is achieved through an opticaltweezing effect. However, phase separation could potentially also be caused by a temperature gradient (such as that generated by the laser, see eqn (19)) through thermophoresis. The onedimensional thermophoresis equation in equilibrium is

$$
\frac{\partial \chi}{\partial t}=D \frac{\partial^{2}}{\partial x^{2}} \chi+D_{\mathrm{T}} \frac{\partial}{\partial x} \chi(1-\chi) \frac{\partial}{\partial x} u(x)=0,
$$
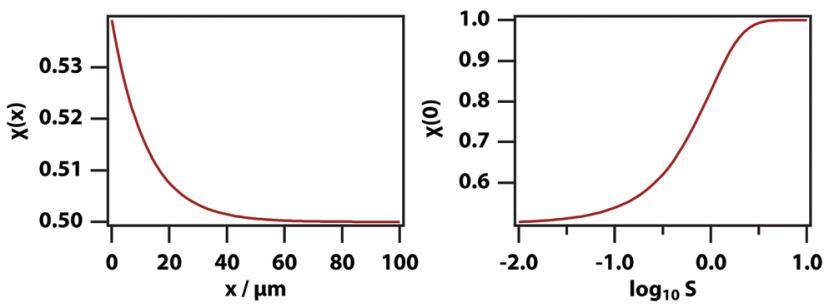

Fig. 5 Thermophoretic effect for $\Delta T=1.6 \mathrm{~K}, \sqrt{ } \alpha / \beta=12.2 \mu \mathrm{m}$, and $c_{0}=0.5$. (left) Mole fraction as a function of position where $x=0$ is the position of the trapping laser focus for $S=10^{-1}$. (right) Mole fraction in the laser focus as a function of $S$.

where $\chi$ is mole fraction, $D$ the diffusion coefficient (as before), and $D_{\mathrm{T}}$ the thermodiffusion coefficient, where the temperature field $u(x)$ caused by the laser is given by eqn (19) and therefore

$$
\frac{\partial}{\partial x} u(x)=\frac{-x}{|x|} \frac{\Delta T}{\sqrt{\alpha / \beta}} \mathrm{e}^{-|x| / \sqrt{\alpha / \beta}} .
$$

Integrating eqn (22) twice over $x$, leads to the solution

$$
\chi(x)=\frac{c_{0} \exp \left(S \Delta T \mathrm{e}^{-x / \sqrt{\alpha / \beta}}\right)}{c_{0} \exp \left(S \Delta T \mathrm{e}^{-x / \sqrt{\alpha / \beta}}\right)+1-c_{0}},
$$

where $c_{0}$ is the initial mole fraction (same as $x_{0}$ as used above but labelled $c_{0}$ to avoid confusion with the position $x$ ), and $S \equiv D_{\mathrm{T}} / D$ is the Soret coefficient. As before, $\sqrt{ } \alpha / \beta$ is the stationary heating spot radius.

Fig. 5 shows the calculated spatially dependent thermophoretic effect as well as the concentration enhancement on the peak. The thermophoretic effect clearly will play a major role for $S>10^{-1}$. For mixtures of small-molecule liquids the Soret coefficient is typically on the order of $S \sim 10^{-3},{ }^{48}$ and therefore thermodiffusion will play no role in the experiments described here.

\section{Methods}

\section{Materials}

Experiments were carried out on nitrobenzene, aniline, decane, hexadecane, cyclohexane, and methylene blue (Sigma Aldrich) and used as supplied. All samples were filtered before use using $0.2 \mathrm{~mm}$ hydrophilic polytetrafluoroethylene (PTFE) filters (Millipore) to remove dust. For all microscopy experiments, a sample thickness of $11.58 \pm 0.19 \mu \mathrm{m}$ was used, controlled by glass monodisperse particle standards (Whitehouse Scientific). Particles were sandwiched between borosilicate glass (VWR) and ruby mica discs, which were cleaned by rinsing in acetone, isopropyl alcohol, and distilled water, followed by drying in an oven at $150{ }^{\circ} \mathrm{C}$ for $30 \mathrm{~min}$. The sample temperature was controlled to $\pm 0.1 \mathrm{~K}$ using a Linkam THMS600 cryogenic microscopy stage. In the experiments, the samples were quenched from room temperature and held at a selected quench temperature. ${ }^{40,41}$

\section{Microscopy}

Microscopy was carried out using an Olympus BX53 light microscope that features modular units for phase-contrast 
and fluorescence microscopy, and a custom unit allowing for simultaneous laser irradiation and microscopy. The primary laser used was a $785 \mathrm{~nm}$ continuous-wave diode laser (Thorlabs) producing a maximum power incident on the sample of $200 \mathrm{~mW}$ with an elliptical mode with a mean beam radius (at half height) of $2.4 \mu \mathrm{m}$ (beam waist $4.1 \mu \mathrm{m}$ ) when using a $\times 10$ objective. Phasecontrast microscopy converts small differences in optical path length into intensity, therefore it can be used as a measure of refractive index. Positive phase contrast has been used here and results in intensity scaling with refractive index for objects on the micrometre scale. Nitrobenzene strongly quenches many fluorescent dyes but the dye methylene blue is quenched relatively weakly. This produces contrast between the nitrobenzene-rich and decanerich phases in fluorescence microscopy. Data were captured using the ImageJ add-on $\mu$ Manager and analysed primarily using ImageJ.

\section{Results}

\section{Laser-induced phase separation (LIPS)}

Experiments were carried out primarily using binary mixtures with a significant difference in refractive index $(\Delta n)$. Mixtures of nitrobenzene and hexadecane were selected for the majority of experiments due to the high $\Delta n\left(1.537^{49}\right.$ and $1.430^{50}$ respectively $)$ and low vapour pressures, in order to maintain consistent mole fractions during sample preparation and long experiments. The mixture is reported to have a bulk (upper consolute) critical temperature $T_{\mathrm{c}}=309.69 \mathrm{~K}=36.54{ }^{\circ} \mathrm{C}$ and critical mole fraction $x_{\mathrm{c}}=0.716 .{ }^{51}$ Our own experiments agree with this figure, with bulk $T_{\mathrm{c}}=36.4 \pm 0.2{ }^{\circ} \mathrm{C}$, however, confinement effects in the $11 \mu \mathrm{m}$ thick microscopy samples elevate the critical temperature to $T_{\mathrm{c}}=37.3 \pm 0.1{ }^{\circ} \mathrm{C}$. An example of an experimentally determined phase diagram is shown in Fig. 6 for nitrobenzenedecane mixtures.

Initial experiments were carried out $0.1^{\circ} \mathrm{C}$ above the binodal at $x_{\mathrm{c}}$. When the $785 \mathrm{~nm}$ laser is focussed in the sample,

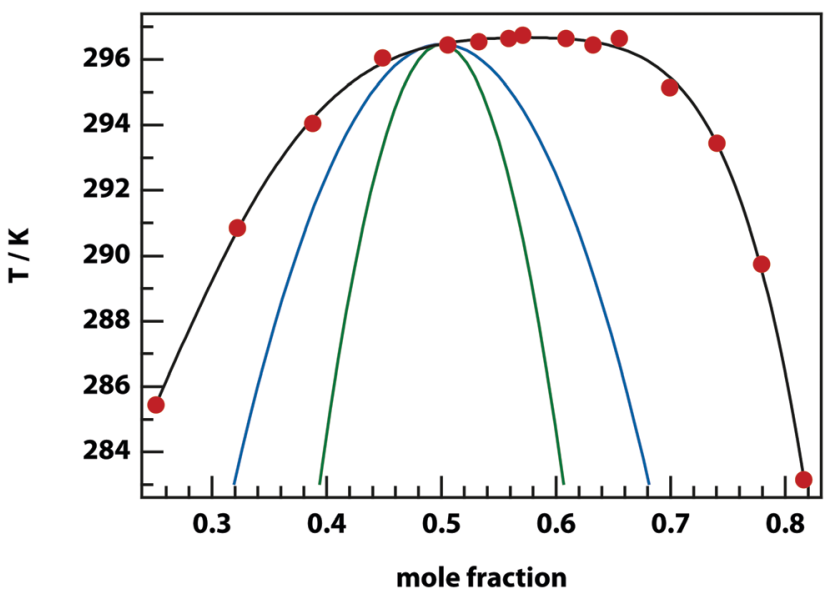

Fig. 6 Phase diagram for nitrobenzene-decane mixtures. Below the experimental binodal curve (black), the mixture separates into two phases that are enriched in either component. Above the binodal, the mixture is a single homogenous phase. Also shown are the theoretical binodal (blue) and spinodal (green) for $\zeta=4.93 \mathrm{~kJ} \mathrm{~mol}^{-1}$.

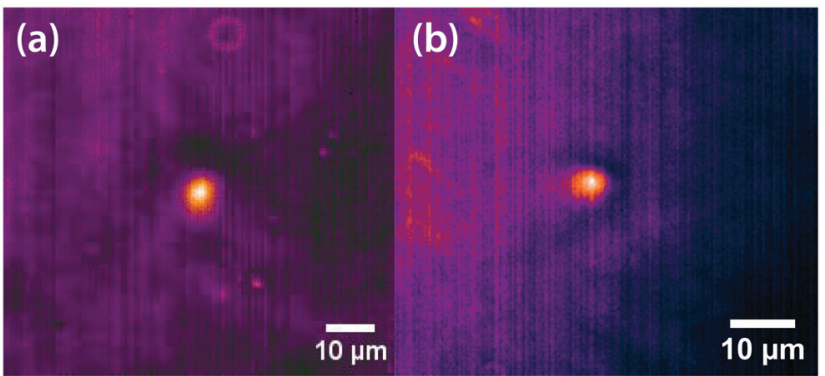

Fig. 7 False coloured phase-contrast microscopy images of LIPS droplets. (a) Nitrobenzene-hexadecane and (b) phenol-decane mixtures. The droplets contain a larger fraction of the high refractive index components, which are nitrobenzene and phenol in this case.

a bright, diffuse droplet becomes visible when viewed using phase-contrast microscopy (Fig. 7). The bright spot indicates that the droplet is composed of a higher fraction of the high refractive-index component than the surrounding liquid.

In addition, a dark region is observed surrounding the droplet, which is nitrobenzene-depleted. This depletion region appears a couple of seconds after the laser is switched on and then equilibrates with the rest of the sample. A radial distribution function (calculated from the image data) of a droplet, which is shown in Fig. 8 gives a full width half maximum (FWHM) of $1.7 \mu \mathrm{m}$ at equilibrium and a depletion region at $7 \mu \mathrm{m}$ after $1.3 \mathrm{~s}$. The minimum of the radial distribution function shifts to larger values as a function of time as predicted by theory (see Fig. 4).

Fluorescence microscopy was carried out on mixtures of nitrobenzene and decane in order to corroborate the finding from phase-contrast microscopy that the droplet in the focus is nitrobenzene-rich. ${ }^{40,41}$ The dye methylene blue was used, as nitrobenzene weakly quenches its fluorescence by electrontransfer, giving good contrast between areas of high and low fractions of nitrobenzene. A dark spot in the focal area confirms that the droplet is composed of a higher fraction of nitrobenzene than the surrounding liquid. ${ }^{40,41}$

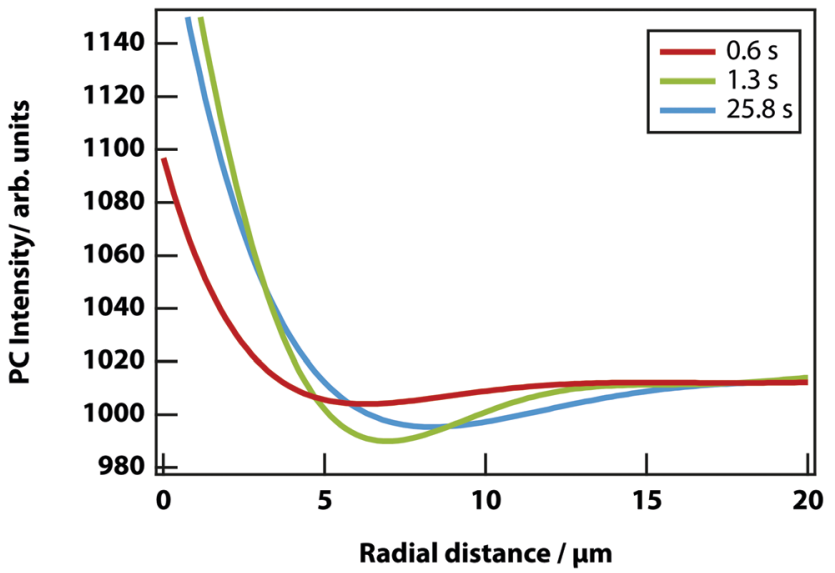

Fig. 8 Radial distribution function of a LIPS droplet as a function of time. It shows the depletion region shifting from 7.0 to $8.3 \mu \mathrm{m}$ from the droplet centre. The region also increases, as the decane fraction reduces and nitrobenzene fraction increases. 
Table 1 List of all mixtures on which LIPS experiments were carried out sorted by refractive index difference $\Delta n$

\begin{tabular}{lll}
\hline Component 1 & Component 2 & $\Delta n$ \\
\hline Nitrobenzene & $n$-Hexane & 0.1812 \\
Aniline & Cyclohexane & 0.1594 \\
Nitrobenzene & $n$-Decane & 0.1452 \\
Nitrobenzene & $n$-Octane & 0.1430 \\
Phenol & $n$-Decane & 0.1190 \\
Nitrobenzene & $n$-Hexadecane & 0.1107 \\
Nitromethane & $n$-Octanol & 0.0355 \\
Nitromethane & $n$-Butanol & 0.0055
\end{tabular}

LIPS has so far been observed in the binary mixtures listed in Table 1, with the exception of the final entry where LIPS was too weak to be detected due to its small $\Delta n$, which is $3 \%$ of the nitrobenzene $n$-hexane mixture. Fig. 9 shows the two mixtures with the smallest $\Delta n$, from which it is clear that nitromethane$n$-octanol is just barely detectable.

Fig. 10 shows the strength of LIPS, as a function of $\Delta n, 0.1^{\circ} \mathrm{C}$ above $T_{\mathrm{c}}$ for the mixtures listed in Table 1 . The effect scales almost as the cube of $\Delta n$. This can be understood based on the quadratic dependence on $\Delta n$ of the optical trap (see eqn (8)) combined with the linear dependence on $\Delta n$ of phase-contrast microscopy.

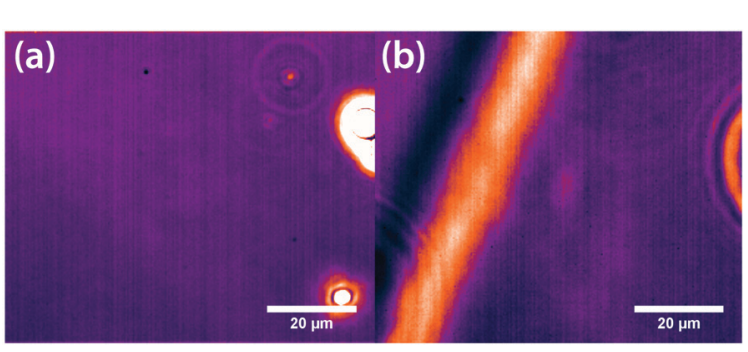

Fig. 9 Mixtures of (a) nitromethane and $n$-butanol with a very small $\Delta n$ of 0.055 and no visible LIPS droplet and (b) nitromethane and $n$-octanol with a small $\Delta n$ of 0.0355 and a faintly visible LIPS droplet in the centre of the image. The other objects in these images are spacer beads and a phase boundary.

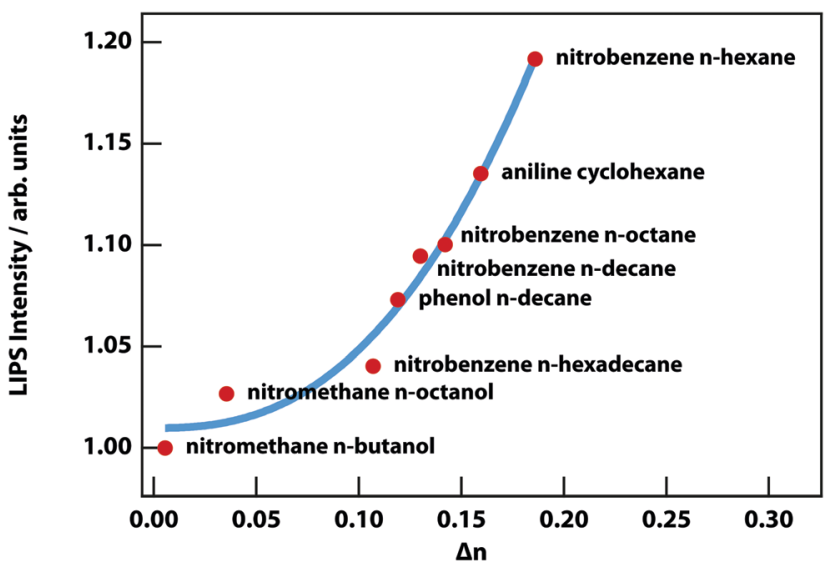

Fig. 10 LIPS intensity as a function of the refractive-index difference. The red circles are the experimentally measured LIPS intensities as a function of the refractive index difference $\Delta n$ measured $0.1 \mathrm{~K}$ above the critical point. The blue solid line is a power-law fit with a power of $2.5 \pm 0.4$.
The dynamics of LIPS was measured by chopping the laser beam and measuring the rise and decay of the LIPS signal (see Fig. 11). The time that it takes for the droplet to form and decay are broadly similar. For different nitrobenzene mixtures (see Table 2), there is no obvious relation between the dynamics and the viscosity (which determines the diffusion rate). However, concomitant changes in $\Delta n$ (and therefore the depth of the optical trapping potential) are likely to mask any effect.

The theory predicts that LIPS follows a power law as a function of the difference in the temperature and the binodal temperature (see Fig. 3). LIPS temperature dependence was measured as a function of temperature above the critical point and is shown in Fig. 12 for two mixtures. These data can be fit to power laws of the form $\left(T-T_{0}\right)^{x}$, where $x$ is -1.0 for nitrobenzene-octane and -2.4 for nitrobenzene-hexadecane broadly consistent with the theoretical prediction. The strength
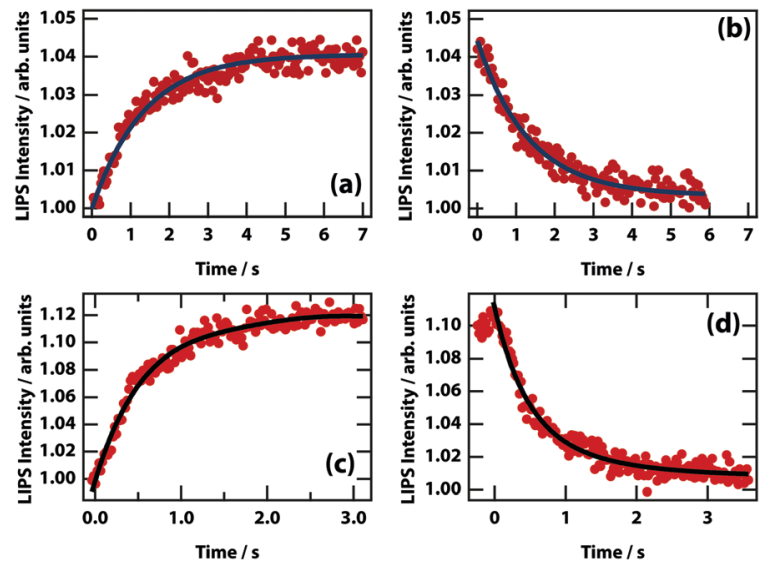

Fig. 11 LIPS formation and decay curves. Dynamics are shown for mixtures of nitrobenzene hexadecane ( $a$ and b) and nitrobenzene octane (c and d). Times at 1/e of the minimum/maximum are listed in Table 2.

Table 2 Formation and decay times of nitrobenzene mixtures with alkanes of different chain length

\begin{tabular}{llll}
\hline Component 2 & Viscosity (cP) & Formation $(\mathrm{s})$ & Decay $(\mathrm{s})$ \\
\hline Octane & 1.10 & 0.60 & 0.87 \\
Decane & 1.31 & 1.31 & 1.68 \\
Hexadecane & 2.48 & 1.19 & 1.11
\end{tabular}
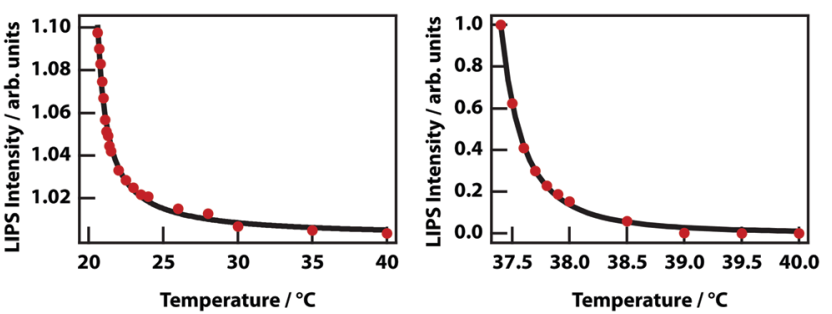

Fig. 12 Temperature dependence of LIPS. (left) Nitrobenzene-octane mixture. As the mixture cools towards $T_{\mathrm{C}}=20.5{ }^{\circ} \mathrm{C}$ the LIPS intensity increases according to a power law with exponent -1.0. (right) A nitrobenzene-hexadecane mixture also fits a power law with exponent -2.4 , and $T_{\mathrm{C}}=37.3^{\circ} \mathrm{C}$. 

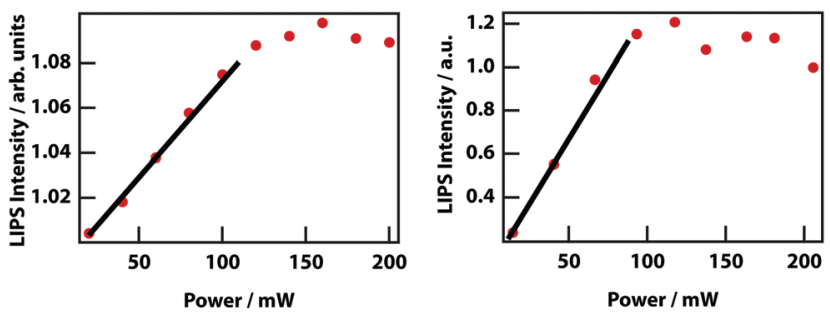

Fig. 13 Power dependence of LIPS. (left) Nitrobenzene-octane and (right) nitrobenzene-hexadecane.
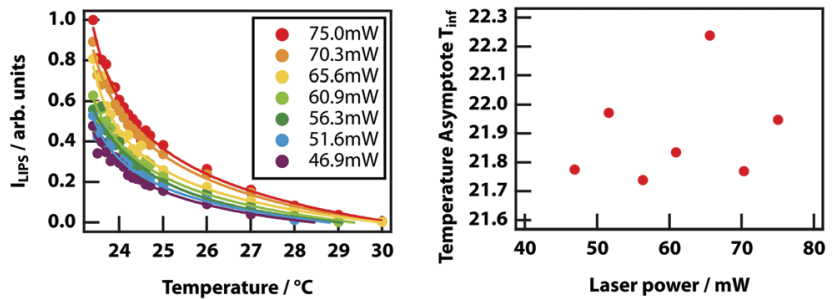

Fig. 14 Power dependence of the temperature dependence of LIPS. (left) Temperature dependence of LIPS measured at seven incident laser powers. Data has been fit to power laws with the exponent fixed at -1 . (right) Temperature asymptotes versus incident laser power. There appear to be no correlation between the two, after fitting to several power law variations.

of LIPS depends not only on the temperature difference relative to $T_{\mathrm{c}}$ but also on the absolute value of the difference in mole fraction relative to $x_{\mathrm{c} \cdot}{ }^{40,41}$

Higher laser power implies a deeper optical trap and therefore enhanced LIPS. Fig. 13 shows that at low powers, there is an approximately linear increase in magnitude of LIPS with power, consistent with eqn (10). At high laser powers and in mixtures with a relatively large $\Delta n$, the droplets become large enough that they are subject to the shade off effect, which is inherent in phase-contrast microscopy, resulting in a saturation of LIPS.

Finally, it was investigated whether the effective critical temperature (temperature asymptote) depends on laser power as predicted by theory (see Fig. 3). Unfortunately, the signal-tonoise ratio of the experiments is insufficient to establish this. Even by fixing the power law exponent to -1 , it was not possible to determine any reasonable trend in the power-dependent temperature asymptotes (see Fig. 14)

\section{LIPS and nucleation (LIPSaN)}

Laser-induced nucleation experiments have a been carried out previously in a variety of metastable (supersaturated) solutions ${ }^{1-5,17,52}$ but a mechanistic understanding of the phenomenon has been lacking. We surmised that laser-induced nucleation was caused by optical trapping near a liquid-liquid critical point. This process would then be laser-induced phase separation and nucleation (LIPSaN).

In the case of liquid-liquid demixing, there is a metastable region between the binodal and spinodal lines. In the metastable region phase separation occurs though a nucleation process identical (in principle) to that of crystal nucleation. Therefore, LIPSaN experiments were attempted on binary mixtures at temperatures and mole fractions in the metastable region. However, it was found that the metastable region is exceedingly narrow, causing spontaneous nucleation and phase separation to occur.

Nevertheless, LIPSaN could be demonstrated in a narrow mole fraction interval $0.62<x<0.69$ for temperatures just above the binodal (see Fig. 15) giving rise to a spinodal decomposition pattern (Fig. 15(b)). Unexpectedly, LIPSaN in these mixtures occurs only once the laser has been switched off rather than on. This can be understood in terms of heating effects. When the tweezing laser is switched on, laser absorption will quickly heat the laser volume and some of the surrounding area (by $\leq 1.6 \mathrm{~K}$, see above; Fig. 16(a)). On a slower timescale (approximately $30 \times$ more slowly, see above), determined by mass diffusion, LIPS will cause the formation of a nitrobenzene enriched droplet surrounded by a depleted volume (shown as a dot and a circle in Fig. 16(b)). When the laser is switched off, both the enriched droplet and the depleted volume will quickly cool (Fig. 16(c)). On a 30× longer timescale equilibrium will be restored (Fig. 16(d)). When such an experiment is carried out near the critical point, nothing happens as all points remain in the mixed region (Fig. 16(e)). When the starting point is at a high mole fraction and near the binodal, the depleted volume will drop into the unstable region below the spinodal when the laser is switched off (Fig. 16(f)). When the starting point is at low mole fraction and near the binodal, the enriched droplet will drop into the unstable region when the laser is switched off (Fig. 16(g)). Phase separation will cause this droplet to further enrich while shrinking at the same time, rendering it invisible because of the lever rule.

Based on the mechanism shown in Fig. 16, the experimental results in Fig. 15 can be understood. Because it is the depleted
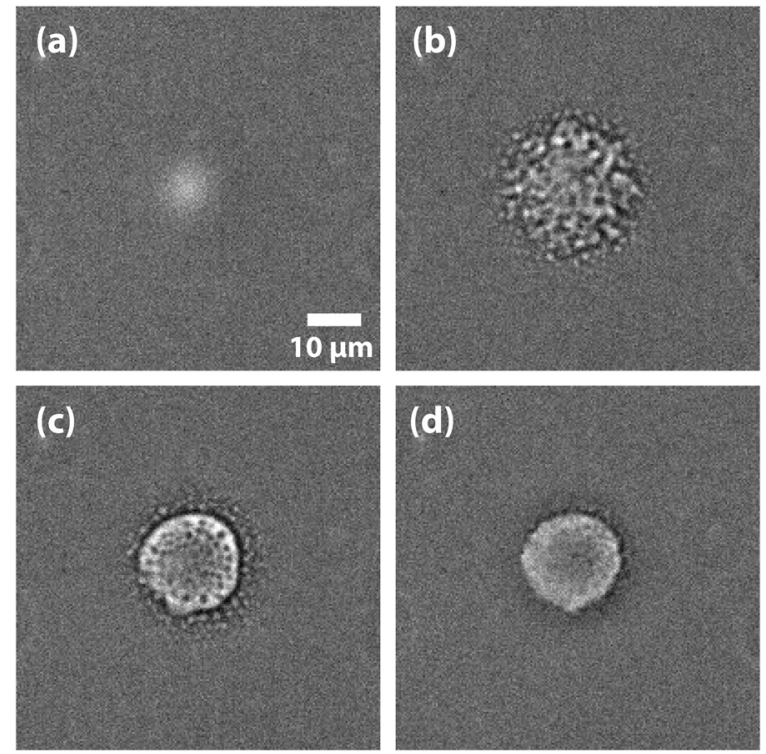

Fig. 15 LIPS and nucleation (LIPSaN) experiments in nitrobenzene-decane. Experiments carried out in a metastable $x=0.632$ nitrobenzene-decane mixture at $T=23.9{ }^{\circ} \mathrm{C}$ with a $120 \mathrm{~mW} 785 \mathrm{~nm}$ focussed laser, on for $30 \mathrm{~s}$. When the laser is switched on a LIPS droplet forms (a) but only once the laser is switched off does nucleation occur (b), followed by Ostwald ripening (c) and (d). ${ }^{40,41}$ 


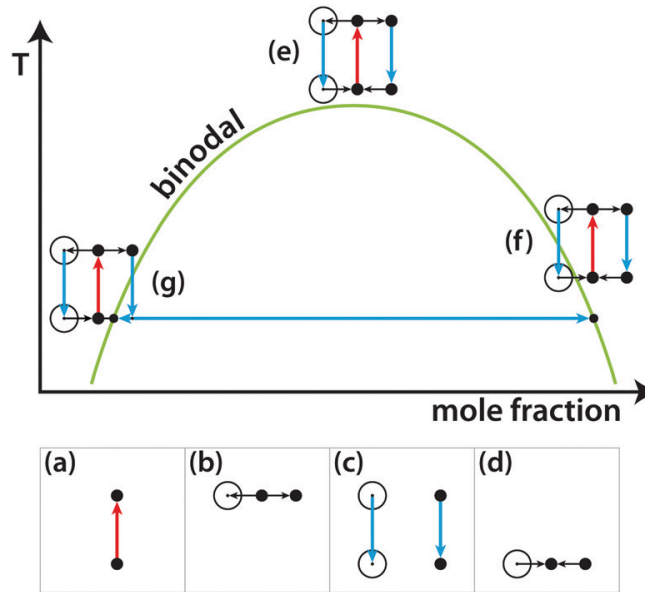

Fig. 16 Schematic liquid-liquid phase diagram showing the combined effects of LIPS and heating. See text for explanation.

region that triggers nucleation, the nucleating region is much larger than the LIPS spot. It also explains why the diameter of the LIPSaN droplet increases with exposure time and laser power. ${ }^{40,41}$

Finally, LIPSaN was investigated as a function of mole fraction. ${ }^{40,41}$ At lower mole fractions (closer to critical, $x=0.575$ ) a large well-defined droplet nucleated, while at higher mole fractions, a cloud of smaller droplets is induced. As can be seen in Fig. 15(c) and (d), Ostwald ripening causes smaller sub-droplets to be subsumed into the main droplet. This effect cannot occur effectively further away from the critical point because of the lower density giving rise to the cloud of smaller droplets.

\section{Discussion and conclusions}

At a phase boundary the free energies of the two phases are identical and fluctuations in concentration or densities can be observed, particularly in the absence of an energy barrier between them. Fluctuations are strongest in proximity to critical points, such as near the upper consolute temperature (UCST) seen in the binary mixtures in this paper. We have demonstrated that these fluctuations can be harnessed using a laser, which generates a trapping potential that draws the high refractive index component of the mixture into the focus. In critical and near-critical mixed samples that are within $\sim 1{ }^{\circ} \mathrm{C}$ of the critical point, this potential forces phase separation. In metastable samples, the laser potential lowers the energy barrier to the separated state and can trigger nucleation.

Photothermal separation has been documented in binary mixtures of molecular liquids ${ }^{53}$ and polymers, ${ }^{44,54,55}$ but relies on the system in question having a lower critical solution temperature (LCST), the opposite of the UCST present in the mixtures we have used. Bunkin et al. used such an LCST mixture and using a model based on the Navier-Stokes equation concluded that heat-induced barodiffusion (diffusion due to pressure) was the dominant mechanism. ${ }^{56}$ Any heating in an LCST system forces the mixture into the separated state, whereas heating in a UCST mixture moves it further into the mixed regime. As such, the mechanism of LIPS cannot be due to a direct heating effect.
One of the main mixture we have used, nitrobenzene-decane, has no electronic absorption bands at the laser wavelength of $785 \mathrm{~nm}$ (see Fig. S1, ESI $\dagger$ ) ruling out the mechanism of resonant trapping of fluorescent molecules. ${ }^{57}$ Thermophoresis, or the Soret effect, is the diffusion of molecules in a mixture due to their preference to be in a region of high or low temperature, lowering the overall free energy. However, using our steady-state focal heating calculation and typical values of the Soret coefficient for small molecules, ${ }^{58}$ we conclude that the change in mole fraction is negligible relative to change due to LIPS.

LIPS is similar to the optical trapping of particles such as glass beads where, due to refraction within the particle, there is a small transfer of momentum that leads to a net restoring force towards the region of highest electric field. ${ }^{59}$ In optical trapping, the scattering force is also important as it pushes the particle out of the focus in the propagation direction of the laser beam. In order to trap particles in bulk liquid, the restoring force must be greater than or equal to the scattering force, which requires an objective lens with a numerical aperture $>\sim 1{ }^{60}$ Our experimental setup requires a long working distance lens, which has a much lower numerical aperture of 0.3. As such in our experiments, the LIPS droplet is pushed against the lower microscope slide and not trapped in the strictest sense.

The LIPS and LIPSaN effects detailed here do not depend on the presence of pre-nucleation clusters that can be trapped and aggregated by the laser. ${ }^{61,62}$ Rather the laser generates a potential that lowers the free energy of the phase-separated state. The mixtures we used were chosen because their critical points are easily accessible and not hidden below a liquidus or above the boiling points of the components. Ultimately, this is a generic effect that applies to all mixtures.

The experiments presented here use LIPS and LIPSaN only to separate two liquids in a mixture near a liquid-liquid critical point. Nearly all liquid mixtures have a UCST although in most cases it will be hidden below the liquidus line. A good example is the aqueous sodium chloride solution, which-even though $\mathrm{NaCl}$ is highly soluble in water-has a hidden binodal and spinodal where an amorphous $\mathrm{NaCl}$ phase separates from water. ${ }^{63}$ The same holds true for aqueous protein solutions. ${ }^{29}$ However, in the case of proteins the position of the fluid-fluid critical point can be manipulated relatively easily using ionic strength and $\mathrm{pH}^{64}$ Some protein solutions under some circumstances will show phase separation into a dense and a dilute-fluid phase and this is even observed in vivo. ${ }^{65,66}$ In cases where the UCST is below the liquidus, LIPS will produce a droplet with an enhanced concentration of one component liquid. This will either greatly increase the probability of nucleation through the greater concentration in a classical Gibbs nucleation process ${ }^{23,35}$ or force the production of a liquid-like droplet that is unstable with respect to crystallisation. ${ }^{67-69}$ Therefore, it is reasonable that LIPS is at the basis of most reported laser-induced crystal nucleation experiments (which would all be a form of LIPSaN).

Thus, LIPS is likely to be related to a string of recent laserinduced nucleation experiments claiming to use optical trapping of clusters, ${ }^{15,17,19,61,62,70-74}$ although these experiments could only be carried out at air-solution interfaces, implying that evaporation, 
Marangoni effects, and thermophoresis may play important roles as well. A repeat of these experiments in the bulk would be worthwhile, bearing in mind the possibility of a hidden liquidliquid critical point enhancing concentration fluctuations. Bulk non-photochemical laser-induced nucleation (NPLIN) phenomena described by Garetz, Alexander, and others ${ }^{1-14,75,76}$ fit with our narrative as the final states have higher refractive indices than their precursor mixtures. There are two potential exceptions to this picture, the first being laser-induced bubble nucleation. Peters and co-workers reported NPLIN of gas bubbles from solutions of carbon dioxide in water. ${ }^{11}$ As a gas, $\mathrm{CO}_{2}$ has a much lower refractive index than water, which would be inconsistent with LIPS. The threshold pulse energy of the effect was reported to be independent of wavelength and purity of the chemicals, suggesting that heating does not play a role. Alexander and coworkers ran similar NPLIN experiments and found that filtering their solutions or thorough cleaning of glassware in acid increased the threshold for NPLIN and decreased the number of nucleation events. ${ }^{77}$ In any case, since our experiments show a depletion region which has a higher fraction of the low refractiveindex component, this may be responsible for lowering the energy barrier to gas-bubble nucleation. The second potential exception to this rule is NPLIN of glacial acetic acid, ${ }^{4}$ as it is a pure liquid rather than a mixture. However, it is well established that pure liquids can exhibit liquid-liquid critical points, as observed in triphenyl phosphite, ${ }^{78-81} n$-butanol, ${ }^{82,83}$ D-mannitol, ${ }^{84}$ and water. ${ }^{85}$ It may be the case that acetic acid exhibits a yet undocumented liquid-liquid critical point.

In summary, we have demonstrated that a cheap laser-diode can be used to induce phase separation and nucleation in nearcritical binary liquid mixtures. The effect depends on both on a difference in refractive index and, more importantly, proximity to a liquid-liquid critical point. The theory presented here applies to laser-induced crystal nucleation and provides a framework for future research on the subject.

\section{Conflicts of interest}

There are no conflicts to declare.

\section{Acknowledgements}

We gratefully acknowledge discussions with Giovanni Volpe (University of Gothenburg) on the possible role of thermophoresis. We thank the Engineering and Physical Sciences Research Council (EPSRC) for support through grants EP/J004790/1, EP/J009733/1, and EP/N007417/1. This work was part funded by the European Research Council (ERC) under the European Union's Horizon 2020 research and innovation program (grant agreement no. 832703).

\section{References}

1 B. A. Garetz, J. E. Aber, N. L. Goddard, R. G. Young and A. S. Myerson, Phys. Rev. Lett., 1996, 77, 3475-3476.

2 B. A. Garetz, J. Matic and A. S. Myerson, Phys. Rev. Lett., 2002, 89, 175501.
3 X. Sun, B. A. Garetz and A. S. Myerson, Growth, 2006, 6, 684-689.

4 M. R. Ward, S. McHugh and A. J. Alexander, Phys. Chem. Chem. Phys., 2012, 14, 90-93.

5 N. Iefuji, R. Murai, M. Maruyama, Y. Takahashi, S. Sugiyama, H. Adachi, H. Matsumura, S. Murakami, T. Inoue, Y. Mori, Y. Koga, K. Takano and S. Kanaya, J. Cryst. Growth, 2011, 318, 741-744.

6 Y. Liu, M. H. van den Berg and A. J. Alexander, Phys. Chem. Chem. Phys., 2017, 19, 19386-19392.

7 C. Duffus, P. J. Camp and A. J. Alexander, J. Am. Chem. Soc., 2009, 131, 11676-11677.

8 W. Li, A. Ikni, P. Scouflaire, X. Shi, N. El Hassan, P. Gémeiner, J. M. Gillet and A. Spasojević-De Biré, Cryst. Growth Des., 2016, 16, 2514-2526.

9 A. Usman, T. Uwada and H. Masuhara, J. Phys. Chem. C, 2011, 115, 11906-11913.

10 T. Kosa, L. Sukhomlinova, L. Su, B. Taheri, T. J. White and T. J. Bunning, Nature, 2012, 485, 347-349.

11 B. C. Knott, J. L. LaRue, A. M. Wodtke, M. F. Doherty and B. Peters, J. Chem. Phys., 2011, 134, 171102.

12 B. C. Knott, N. Duff, M. F. Doherty and B. Peters, J. Chem. Phys., 2009, 131, 224112.

13 B. C. Knott, M. F. Doherty and B. Peters, J. Chem. Phys., 2011, 134, 154501.

14 Y. Liu, M. R. Ward and A. J. Alexander, Phys. Chem. Chem. Phys., 2017, 19, 3464-3467.

15 K. I. Yuyama, T. Rungsimanon, T. Sugiyama and H. Masuhara, Cryst. Growth Des., 2012, 12, 2427-2434.

16 T. Sugiyama, K. Yuyama and H. Masuhara, Acc. Chem. Res., 2012, 45, 1946-1954.

17 K. Yuyama, T. Sugiyama and H. Masuhara, J. Phys. Chem. Lett., 2013, 4, 2436-2440.

18 K. I. Yuyama, M. Ueda, S. Nagao, S. Hirota, T. Sugiyama and H. Masuhara, Angew. Chem., Int. Ed., 2017, 56, 6739-6743.

19 H. Niinomi, T. Sugiyama, K. Miyamoto and T. Omatsu, Cryst. Growth Des., 2018, 18, 734-741.

20 R. W. Bowman and M. J. Padgett, Rep. Prog. Phys., 2013, 76, 26401.

21 S. Bartkiewicz and A. Miniewicz, Phys. Chem. Chem. Phys., 2015, 17, 1077-1083.

22 D. Gebauer and H. Coelfen, Nano Today, 2011, 6, 564-584.

23 R. J. Davey, S. L. M. Schroeder and J. H. Ter Horst, Angew. Chem., Int. Ed., 2013, 52, 2166-2179.

24 H. Gebauer, D. Völkel and A. Cölfen, Science, 2008, 322, 1819-1822.

25 G. De With and N. A. J. M. Sommerdijk, Science, 2009, 587, 2007-2010.

26 A. Dey, P. H. H. Bomans, F. A. Müller, J. Will, P. M. Frederik, G. De With and N. A. J. M. Sommerdijk, Nat. Mater., 2010, 9, 1010-1014.

27 P. J. M. Smeets, A. R. Finney, W. J. E. M. Habraken, F. Nudelman, H. Friedrich, J. Laven, J. J. De Yoreo, P. M. Rodger and N. A. J. M. Sommerdijk, Proc. Natl. Acad. Sci. U. S. A., 2017, 201700342.

28 A. E. S. Van Driessche, N. Van Gerven, P. H. H. Bomans, R. R. M. Joosten, H. Friedrich, D. Gil-Carton, N. A. J. M. Sommerdijk and M. Sleutel, Nature, 2018, 556, 89-94. 
29 P. R. ten Wolde and D. Frenkel, Science, 1997, 277, 1975-1978.

30 J. Wedekind, L. Xu, S. V. Buldyrev, H. E. Stanley, D. Reguera and G. Franzese, Sci. Rep., 2015, 5, 1-7.

31 L. Xu, S. V. Buldyrev, H. E. Stanley and G. Franzese, Phys. Rev. Lett., 2012, 109, 095702.

32 P. E. Bonnett, K. J. Carpenter, S. Dawson and R. J. Davey, Chem. Commun., 2003, 698-699.

33 M. Takasuga and H. Ooshima, Cryst. Growth Des., 2014, 14, 6006-6011.

34 J. Mosses, D. A. Turton, L. Lue, J. Sefcik and K. Wynne, Chem. Commun., 2014, 50, 1-9.

35 D. Gebauer, M. Kellermeier, J. D. Gale, L. Bergström and H. Cölfen, Chem. Soc. Rev., 2014, 43, 2348-2371.

36 A. F. Wallace, L. O. Hedges, A. Fernandez-martinez, P. Raiteri, J. D. Gale, G. A. Waychunas, S. Whitelam, J. F. Banfield and J. J. De Yoreo, Science, 2013, 692, 885-889.

37 A. Casner and J.-P. Delville, Phys. Rev. Lett., 2001, 87, 054503.

38 R. D. Schroll, R. Wunenburger, A. Casner, W. W. Zhang and J. P. Delville, Phys. Rev. Lett., 2007, 98, 1-4.

39 Y. Katsir and Y. Tsori, J. Phys.: Condens. Matter, 2017, 29, 63002.

40 F. Walton and K. Wynne, Nat. Chem., 2018, 10, 506-510.

41 F. Walton and K. Wynne, Proc. SPIE, 2018, 10723, 1072300.

42 R. A. L. Jones, Soft Condensed Matter, Oxford Univ. Press Inc., New York, 2002.

43 H. Toyama, K. Yoshikawa and H. Kitahata, Phys. Rev. E: Stat., Nonlinear, Soft Matter Phys., 2008, 78, 1-4.

44 J. P. Delville, C. Lalaude and A. Ducasse, Phys. A, 1999, 262, 40-68.

45 P. F. Barbara, E. Aakesson, G. C. Walker, A. E. Johnson and N. E. Levinger, J. Phys. Chem., 2005, 96, 3728-3736.

46 M. L. Cordero, E. Verneuil, F. Gallaire and C. N. Baroud, Phys. Rev. E: Stat., Nonlinear, Soft Matter Phys., 2009, 79, 011201.

47 M. Benitez, Opt. Eng., 2018, 48, 1-8.

48 M. Eslamian and M. Z. Saghir, Phys. Rev. E: Stat., Nonlinear, Soft Matter Phys., 2009, 80, 1-11.

49 S. Kedenburg, M. Vieweg, T. Gissibl and H. Giessen, Opt. Mater. Express, 2012, 2, 1588-1611.

50 D. L. Camin, A. F. Forziati and F. D. Rossini, J. Phys. Chem., 1954, 58, 440-442.

51 J. Wang, PhD thesis, Univ. Maryl., 2006.

52 M. R. Ward and A. J. Alexander, Cryst. Growth Des., 2012, 12, 4554-4561.

53 S.-A. Mukai, N. Magome, H. Kitahata and K. Yoshikawa, Appl. Phys. Lett., 2003, 83, 2557-2559.

54 C. Crauste-thibierge, A. Petrosyan and S. Ciliberto, Phys. Rev. E: Stat., Nonlinear, Soft Matter Phys., 2015, 92, 052312.

55 J. Hofkens, J. Hotta, K. Sasaki, H. Masuhara and K. Iwai, Langmuir, 2002, 13, 414-419.

56 N. F. Bunkin, A. V. Lobeev, G. A. Lyakhov and Y. P. Svirko, Kvantovaya Elektron., 1996, 23, 62-66.

57 M. A. Osborne, S. Balasubramanian, W. S. Furey and D. Klenerman, J. Phys. Chem. B, 1998, 102, 3160-3167.

58 S. Wiegand, J. Phys.: Condens. Matter, 2004, 16, R357-R379.
59 S. E. S. Spesyvtseva and K. Dholakia, ACS Photonics, 2016, 3, 719-736.

60 M. Woerdemann, in Structured Light Fields, ed. M. Wördemann, Springer Berlin Heidelberg, Berlin, Heidelberg, 2012, pp. 5-26.

61 C. S. Wu, P. Y. Hsieh, K. I. Yuyama, H. Masuhara and T. Sugiyama, Cryst. Growth Des., 2018, 18, 5417-5425.

62 K. Yuyama, M. J. Islam, K. Takahashi, T. Nakamura and V. Biju, Angew. Chem., Int. Ed., 2018, 57, 13424-13428.

63 H. Jiang, P. G. Debenedetti and A. Z. Panagiotopoulos, J. Chem. Phys., 2019, 150, 1-23.

64 Y. Wang, A. Lomakin, S. Kanai, R. Alex and G. B. Benedek, Langmuir, 2017, 33, 7715-7721.

65 A. A. Hyman, C. A. Weber and F. Jülicher, Annu. Rev. Cell Dev. Biol., 2014, 30, 39-58.

66 H. Le Ferrand, M. Duchamp, B. Gabryelczyk, H. Cai and A. Miserez, J. Am. Chem. Soc., 2019, 141, 7202-7210.

67 J. F. Lutsko, Sci. Adv., 2019, 5, eaav7399.

68 D. Gebauer and S. E. Wolf, J. Am. Chem. Soc., 2019, 141, 4490-4504.

69 A. Ianiro, H. Wu, M. M. J. van Rijt, M. P. Vena, A. D. A. Keizer, A. C. C. Esteves, R. Tuinier, H. Friedrich, N. A. J. M. Sommerdijk and J. P. Patterson, Nat. Chem., 2019, 11, 320-328.

70 J. R. Tu, A. Miura, K. I. Yuyama, H. Masuhara and T. Sugiyama, Cryst. Growth Des., 2014, 14, 15-22.

71 K. Yuyama, J. George, K. G. Thomas, T. Sugiyama and H. Masuhara, Cryst. Growth Des., 2016, 16, 953-960.

72 H. Niinomi, T. Sugiyama, M. Tagawa, K. Murayama, S. Harada and T. Ujihara, CrystEngComm, 2016, 18, 7441-7448.

73 K. Yuyama, K.-D. Chang, J.-R. Tu, H. Masuhara and T. Sugiyama, Phys. Chem. Chem. Phys., 2018, 20, 6034-6039.

74 H. Niinomi, T. Sugiyama, S. Uda, M. Tagawa, T. Ujihara, K. Miyamoto and T. Omatsu, Cryst. Growth Des., 2019, 19, 529-537.

75 T. Tasnim, A. Goh, O. Gowayed, C. T. Hu, T. Y. Chen, J. E. Aber and B. A. Garetz, Cryst. Growth Des., 2018, 18, 5927-5933.

76 A. J. Alexander and P. J. Camp, J. Chem. Phys., 2019, 150, 040901.

77 M. R. Ward, W. J. Jamieson, C. A. Leckey and A. J. Alexander, J. Chem. Phys., 2015, 142, 144501.

78 J. Mosses, C. D. Syme and K. Wynne, J. Phys. Chem. Lett., 2014, 6, 38-43.

79 K. Murata and H. Tanaka, Proc. Natl. Acad. Sci. U. S. A., 2019, 116, 7176-7185.

80 M. Kobayashi and H. Tanaka, Nat. Commun., 2016, 7, 1-8.

81 F. Walton, J. Bolling, A. Farrell, J. MacEwen, C. Syme, M. G. Jiménez, H. M. Senn, C. Wilson, G. Cinque and K. Wynne, DOI: 10.26434/chemrxiv.9891491.v1.

82 C. D. Syme, J. Mosses, M. González-Jiménez, O. Shebanova, F. Walton and K. Wynne, Sci. Rep., 2017, 7, 42439.

83 R. Kurita and H. Tanaka, J. Phys.: Condens. Matter, 2005, 17, L293-L302.

84 M. Zhu, J. Q. Wang, J. H. Perepezko and L. Yu, J. Chem. Phys., 2015, 142, 244504.

85 S. Woutersen, B. Ensing, M. Hilbers, Z. Zhao and C. A. Angell, Science, 2018, 359, 1127-1131. 